Revista de Economia Política, vol. 42, no 1, pp. 88-104, janeiro-março/2022

\title{
Restrição de sobrevivência e regulação financeira: uma nova perspectiva minskyana
}

\author{
Survival constraint and financial regulation: \\ a new Minskyian approach
}

ERNANI TEIXEIRA TORRES FILHO*
NORBERTO MONTANI MARTINS**

RESUMO: Este artigo aponta a importância do conceito de "restrição de sobrevivência" proposto por Minsky na gênese da introdução da regulação financeira moderna. O risco de colapsos sistêmicos nos sistemas baseados em moeda fracionários levou os Estados a suspenderem a aplicação da penalidade relacionada à restrição de sobrevivência - a falência - aos bancos comerciais. Essa medida permitiu que esses agentes financeiros pudessem, na busca por lucros, tomar decisões alocativas ainda mais arriscadas, aumentando a fragilização financeira (moral hazard). Para mitigar esse comportamento adverso, a regulação financeira procura todo o tempo estabelecer limites que emulem o comportamento que esses agentes deveriam ter caso ainda estivessem sujeitos à restrição de sobrevivência. Essas regras precisam ser permanentemente revistas de modo a acompanhar a evolução dos mercados financeiros sob pena de se tornarem impotentes para evitar situações de instabilidade financeira

PALAVRAS-CHAVE: Minsky; restrição de sobrevivência; sistema financeiro; regulação financeira; instabilidade financeira.

ABSTRACT: This article points out the importance of Minsky's concept of 'survival constraint' in the genesis of financial regulation. The risk of a systemic collapse led the States to suspend the enforcement of the penalty related to the survival constraint - bankruptcy - on commercial banks. This suspension allowed relevant financial agents, in the pursuit of profits, to make even riskier allocative decisions (moral hazard), increasing the level of financial fragility. To mitigate this moral behavior, governments introduced financial regulation, fixing the limits that should emulate the behavior of these agents if they were still subject to the

\footnotetext{
* Instituto de Economia da Universidade Federal do Rio de Janeiro - IE/UFRJ, Rio de Janeiro/RJ, Brasil. E-mail: ernanit@hotmail.com. Orcid: https://orcid.org/0000-0001-9588-5769.

* Instituto de Economia da Universidade Federal do Rio de Janeiro - IE/UFRJ, Rio de Janeiro/RJ, Brasil. E-mail: norberto.martins@ie.ufrj.br. Orcid: https://orcid.org/0000-0002-9101-3135. Submetido: 4/Maio/2020; Aprovado: 10/Fevereiro/2021.
} 
survival constraint. These rules must be permanently revisited to keep up with the evolution of the financial markets or they will become unable to avoid financial instability.

KEYWORDS: Minsky; survival constraint; financial system; financial regulation; financial instability.

JEL Classification: G00; G01; G18; G21; G28.

\section{INTRODUÇÃO}

Hyman Minsky é um economista ainda pouco conhecido. Sua obra, no entanto, adquire alguma notoriedade nos eventos de crises financeiras. Nessas ocasiões, a Hipótese da instabilidade Financeira (HIF) é saudada como a teoria que consegue explicar por que os mercados financeiros são capazes de gerar recessões fortes e prolongadas. Alguns analistas chegaram a denominar a crise de 2008 como um "momento Minsky" (Cassidy, 2008), mas, tão logo a recessão foi superada, novamente, se reduziu o interesse pela obra do autor.

Minsky tinha uma visão muito particular de como o sistema de mercado funciona: "A capitalist economy can be described by a set of interrelated balance sheets and income statements" (Minsky, 1992a:12). Para ele, o capitalismo é essencialmente um sistema financeiro e a dinâmica econômica gira ao redor dos impactos que as finanças têm sobre o comportamento dos atores econômicos e o sistema como um todo (Minsky, 1967:33). Nesse ambiente, todas as unidades econômicas precisam gerenciar fluxos de caixa e balanços de modo que a quantidade de dinheiro sob seu comando seja sempre igual ou maior que suas despesas correntes. Caso contrário, terão sua própria sobrevivência ameaçada (Minsky, 2005). Essa noção de "restrição de sobrevivência" é pouco explorada pela literatura. Entretanto, conceitos minskyanos mais conhecidos como a fragilidade financeira e a hipótese de instabilidade financeira guardarem importante relação com a survival constraint.

Vários autores pós-keynesianos utilizaram elementos do pensamento minskyano para problematizar a questão da regulação financeira (Sobreira, 2005; Freitas, 2010; Hermann e Paula, 2014; Carvalho, 2015). Essa literatura frequentemente associa o tema à incerteza e à noção de funcionalidade ${ }^{1}$ do sistema financeiro, adicionando uma "dimensão minskyana" à questão da alocação e da geração de recursos pelo sistema financeiro. Compreende a regulação como uma forma de intervenção corretiva do Estado sobre os mercados.

Este artigo busca preencher uma lacuna nessa literatura. Propomos uma visão alternativa que integre três elementos relevantes no pensamento de Minsky: restrição de sobrevivência, instabilidade e regulação financeira. Para tanto, promovemos uma revisão de seus escritos, disponibilizados pelo Levy Economics Institute of

\footnotetext{
${ }^{1} \mathrm{O}$ conceito de funcionalidade é uma alternativa pós-keynesiana ao conceito de eficiência (Studart, 1995).
} 
Bard College, e dos trabalhos de autores que buscaram interpretar o pensamento de Minsky, como Kregel (2014) e Wray (2017).

Além desta introdução, o artigo conta com mais cinco seções. A primeira apresenta os conceitos de restrição de sobrevivência e de margens de segurança. A segunda discute a noção de fragilidade financeira e apresenta a Hipótese de Instabilidade Financeira. A terceira discute a regulação sob a ótica minskyana. A quarta seção trata dos princípios norteadores das políticas regulatórias, desde uma perspectiva minskyana. Por fim, as considerações finais consolidam os principais pontos do artigo.

\section{RESTRIÇÃO DE SOBREVIVÊNCIA E MARGENS DE SEGURANÇA}

Minsky introduziu em 1954, em sua tese de doutorado, o conceito de survival constraint ou restrição de sobrevivência (Mehrling, 1999; Nielson, 2019; Torres Filho, 2019). Segundo sua definição:

Survival conditions are an effective constraint upon the behavior of firms. [...] Survival conditions have been defined as requiring that total money expenses be less than or equal to total money receipts (ignoring whatever initial liquidity the firm possesses) for every time period from the initial position to the firm's horizon. The objective phenomenon related to the survival of a firm is its balance sheet structure. (Minsky, 2005:127)

Trata-se, portanto, de uma obrigação permanente, a que os agentes econômicos, à exceção do banco central, estão submetidos pelo Estado. Por essa norma, todos precisam a qualquer tempo realizar pagamentos na moeda imposta pelo poder público. A penalidade para quem seja flagrado transgredindo essa limitação pode comprometer a existência do infrator. O termo "sobrevivência" aparece nesse contexto com um duplo significado. Para as empresas, trata-se de evitar a falência - ou no mínimo o embaraço de estar sob alguma forma de execução judicial. Para as famílias, está relacionada às dificuldades associadas ao provimento de suas necessidades básicas. Diante dessa imposição, empresas, famílias, órgãos de governo e bancos não têm alternativa senão comandar a todo momento uma soma de dinheiro grande o suficiente para fazer frente a seus gastos correntes.

A limitação imposta pela moeda é no dia a dia administrada através de diferentes mercados e mecanismos de liquidez. Isto acontece porque os agentes econômicos:

often make payment commitments without having the cash on hand. Almost always, the expectation is that the cash flow from operations will be large enough to cover the commitment. However, if operations do not generate a sufficiently large cash flow, then the survival of the unit - or at least the avoidance of embarrassment - requires that the unit be able to borrow or sell assets to obtain the necessary cash. The extent to which savings intermediaries can be liquid, and to which firms can 
make commitments in advance of funding, depends upon the scope and strength of money market intermediation. The money market enables organizations to be safely illiquid - provided they have assets, which are eligible for this market. The basic raw material for the money market is the existence of pools of excess of liquidity. (Minsky, 1967:33)

A preocupação com a liquidez, a existência de instrumentos e de mercados para administrá-la e, como veremos, a presença da regulação financeira são decorrências da forma como a restrição de sobrevivência é gerida nas sociedades modernas. Isso faz com que essa limitação seja tão importante nas estratégias e nas decisões das firmas quanto a maximização de lucro: "Business firms may be considered to be as interested in surviving as in maximizing profit" (Minsky, 2005:152). Desse ponto de vista, os sistemas monetários e financeiros modernos existem fundamentalmente para permitir que os agentes gerenciem com flexibilidade seus fluxos de caixa. A capacidade de precificar o risco de crédito desses atores abre possibilidade de ganhos que permitem o aparecimento de mercados para transações com esses ativos ou de prover garantia contra a volatilidade de seus valores (derivativos).

Além de ter um papel determinante no comportamento dos agentes, a restrição de sobrevivência também possui uma dimensão macroeconômica. A cada momento, as unidades econômicas que não emitem moeda (ANE) estão coletivamente subordinadas a uma limitação global imposta pela quantidade de moeda existente na economia. O aumento ou a redação na disponibilidade de dinheiro se traduzirá imediatamente no relaxamento ou no estreitamento da restrição de sobrevivência desses atores.

Em um sistema moderno, o montante de depósitos à vista - a principal forma da moeda moderna - flutua e é estabelecido a partir das operações de crédito entre os ANE e os bancos emissores. Estas instituições, por sua vez, estão também sujeitas à restrição de sobrevivência, só que em uma moeda diferente do resto da sociedade. Os bancos, diferentemente, são obrigados a liquidar seus pagamentos por meio dos depósitos (reservas) que precisam manter junto à autoridade monetária. Há assim uma hierarquia no sistema monetário, relacionada à administração dessas flexibilizações por cada tipo de agente (Torres Filho, 2019).

Normalmente, a criação e a destruição de moeda no primeiro nível da hierarquia ocorrem de forma relativamente automática, dado a demanda dos ANE, a política de crédito dos bancos e a política monetária do banco central. Entretanto, há ocasiões em que as instituições emissoras de depósitos à vista deixam de se sentir confortáveis em validar as solicitações de seus clientes, por acharem que essa estratégia estaria colocando em risco sua própria restrição de sobrevivência junto ao banco central. Nesses casos, se tornam mais seletivas e aumentam as taxas de juros e as exigências de garantias. Outra causa dessa mudança de comportamento pode ocorrer se o banco central unilateralmente aumentar a taxa de juros básica da economia, pressionando a restrição de sobrevivência do conjunto dos bancos. Essa medida, em princípio, deve levá-los a adotar uma postura mais conservadora em suas concessões de crédito. Em qualquer desses cenários, haverá sempre um 
aumento imediato nos limites impostos pela restrição de sobrevivência aos ANE que dependem de crédito para equilibrar seus fluxos de caixa.

Para se defenderem de situações como essas, os agentes econômicos e os bancos se utilizam de alguns mecanismos de proteção, as chamadas "margens de segurança" (margins of safety). Como afirmou Minsky (1991):

A fundamental property of all capitalist economies is the existence of a system of borrowing and lending based upon various margins of safety. The excess of anticipated cash flows from asset ownership or participation in income production over the cash flows committed by the liability structure is one class of margins of safety. The excess of the market or the pledge value of assets over the value of liabilities which can require the payment of some principle amount is another class of margins of safety" (Minsky, 1992a:12)

Em outra passagem, o autor aprofunda esse entendimento propondo uma tipologia de margens de segurança (Minsky, 1977a:17). Wray (2017) aprofunda esse insight de Minsky e propõe a existência de três tipos de margem de segurança. O principal deles é o diferencial entre a receita futura esperada com algum grau de certeza e os compromissos assumidos ou projetados para o mesmo período. É o "colchão de fluxo de caixa" (cash-flow cushion). Quanto maior for essa folga, mais robusta financeiramente é a unidade econômica. Essa racionalidade econômica vale tanto para famílias e empresas quanto para bancos.

Um segundo tipo são as reservas líquidas acumuladas pela unidade, o "colchão de liquidez" (liquidity cushion), na forma de dinheiro ou de ativos de baixa volatilidade de preço e que sejam facilmente vendáveis ou dados em garantia. Entretanto, quanto mais difícil for transformar esse segundo tipo de margem de segurança em dinheiro, menos potentes elas serão. Finalmente, o terceiro tipo de margem de segurança está associado à solidez do balanço da unidade (balance-sheet cushion). Diz respeito, portanto, à sua capacidade de tomar crédito sem oferecer garantias. Esses casos se restringem aos agentes que acumulam um volume muito elevado dos outros tipos de margens de segurança (Wray, 2017:99-100). As margens de segurança são, assim, mecanismo voltados para a administração que cada unidade está obrigada a fazer da sua própria restrição de sobrevivência.

O cálculo do tamanho apropriado e da composição entre esses diferentes instrumentos de garantia pode mudar abruptamente, já que está sujeito à incerteza e depende do estado de confiança. Assim, o volume e a relevância de cada tipo de margem de segurança mudam ao longo do ciclo financeiro reduzindo-se relativamente quanto mais se prolonga um período de estabilidade. Em compensação, tendem a se elevar substancialmente em momentos de pânico ou crise financeira. 


\section{FRAGILIDADE E INSTABILIDADE FINANCEIRAS}

A HIF deve ser entendida como uma consequência da restrição de sobrevivência em um contexto em que a busca do lucro se dá em um ambiente onde já existe um mercado financeiro desenvolvido. Em uma economia com essas características, os atores econômicos administram essa limitação como parte de uma estratégia de geração de lucros. Para alguns credores e devedores, conviver com margens de segurança menores em momentos de liquidez abundante é uma opção tentadora de rentabilização. Para outros, pode até mesmo se tornar uma característica permanente de investimentos financeiros muito arriscados, a exemplo do que aconteceu nos mercados de seguros e de derivativos nos EUA antes da crise de $2008^{2}$.

Para Minsky, o sistema monetário moderno apresenta uma tendência inexorável ao risco crescente, pelo fato de a liquidez ser administrada privadamente, ou seja, como parte de uma estratégia de geração de lucros dos bancos. Assim, ao longo do tempo, novas estruturas e produtos financeiros vão tomando o lugar de operações financeiramente mais robustas, nas quais os agentes, em tempos normais, seriam até mesmo capazes de saldar todas suas dívidas apenas com sua geração corrente de caixa. Esse tipo de postura foi chamado por Minsky de hedge.

Em vez disso, as empresas e as famílias se tornam cada vez mais dependentes da captação de recursos provenientes do mercado financeiro para obterem rotineiramente o dinheiro necessário para equilibrar seus fluxos de caixa. Isso acontece porque os bancos, da sua parte, passam a conceder empréstimos com base em uma expectativa de receitas operacionais líquidas de seus clientes insuficientes para garantir o pagamento da dívida. Portanto, credores e devedores sabem de antemão que essas dívidas precisarão ser refinanciadas antes do fim do prazo contratado. Essa nova postura faz com que empresas e seus bancos fiquem sujeitos ao risco relacionado às condições de preço e prazo prevalecentes no mercado financeiro no momento em que essa renegociação vier a se dar. Essa estratégia de financiamento foi denominada por Minsky de "especulativa".

Na medida em que o período de estabilidade financeira se prolonga, esse comportamento especulativo se generaliza e intensifica. Nessa fase, as unidades econômicas trabalham com uma quase certeza de que a gestão especulativa de sua restrição de sobrevivência será segura, graças à operação cada vez mais “eficaz” dos mercados de liquidez e de crédito. Cada vez mais créditos sujeitos à renegociação são contratados, comprometendo a robustez dos balanços desses agentes. Nesse ambiente, surgem unidades nas quais as receitas correntes são insuficientes para liquidar não só as parcelas de amortização, mas também parte dos juros, o que provoca o aumento contínuo do seu endividamento. Esse grupo de empresas muito especulativas foi chamado por Minsky de "Ponzi".

Assim, em períodos de prolongada prosperidade, há uma tendência à fragilização das estruturas e, à medida que o tempo passa, maiores são as chances de que

\footnotetext{
${ }^{2}$ Ver os exemplos do Lehman Brothers e da AIG em Tooze (2018).
} 
eventuais flutuações no nível de atividade se transformem em crises financeiras de porte. Caso irrompa uma crise e a liquidez venha a se evaporar, a reestruturação de passivos de unidades especulativas - e, principalmente, das unidades Ponzi - poderá não chegar a bom termo, comprometendo a própria sobrevivência desses devedores e realimentando o processo de crise. Este é o cerne da Hipótese da Instabilidade Financeira (Minsky, 1992b).

\section{O PAPEL DA RESTRIÇÃO DE SOBREVIVÊNCIA NA REGULAÇÃO FINANCEIRA}

Nos sistemas econômicos modernos, a quantidade de moeda é administrada por instituições financeiras privadas em um ambiente hierarquizado e sujeito a inovações, acirrada competição, forte volatilidade e incerteza. Neste contexto, Minsky enxerga a regulação financeira como uma atividade essencial, que tem nos bancos comerciais o seu foco principal: "In order to contain the destabilizing effect of banking, it is necessary to regulate" (Minsky, 2008:356).

Os bancos são instituições particularmente importantes para a regulação porque emitem moeda e administram o sistema de pagamentos. Ao conceder empréstimos sem precisar acumular dinheiro antecipadamente, tornam-se o principal instrumento de flexibilidade monetária. Quando um banco concede um empréstimo, está comprando um fluxo de caixa futuro de outro agente em troca de dinheiro hoje. Ao mesmo tempo, o devedor obtém uma entrada imediata de dinheiro contra uma obrigação de pagamento futuro de juros e amortização. O banco garante, assim, a credibilidade do devedor e, consequentemente, valida os passivos de seu cliente.

Para fornecer serviços de liquidez com segurança e gerar lucros, os bancos comerciais deveriam se preocupar com o fato de o fluxo de caixa e outras margens de segurança (e.g., garantias) de seus clientes serem suficientes para honrar suas dívidas. Essa avaliação os protege do risco de liquidez, já que os depositantes podem resgatar seus depósitos à vista a qualquer momento ou o próprio banco pode encontrar restrições para se financiar.

Para Minsky, se não houvesse regulação e suporte financeiro dos governos, os bancos operariam de maneira muito conservadora (Minsky, 1959). Nessa situação hipotética, enfrentariam severas restrições de liquidez (capacidade de pagar dívidas no vencimento) e solvência (existência contínua de patrimônio líquido positivo). Os bancos são, por natureza, empresas altamente alavancadas que adquirem ativos mediante a emissão de passivos de curtíssimo prazo. Assim, precisam manter saldos suficientes para enfrentar perdas inesperadas no sistema de compensação. Como qualquer outra unidade econômica, têm que gerenciar sua restrição de sobrevivência. Entretanto, diferentemente dos demais agentes, estão muito expostos a qualquer queda significativa no valor dos ativos que possuem (dívidas de seus clientes) já que operam com um capital próprio pequeno em comparação ao volume de ativos que administram. Essas limitações, por si sós, deveriam ser suficientes para fazer com que os banqueiros adotassem políticas conservadoras. 
Esse comportamento não é, no entanto, suficiente para neutralizar os efeitos da concorrência que empurram o sistema econômico na direção de aumentar a fragilidade financeira. Como os bancos administram o sistema de pagamentos - portanto, todos os fluxos de caixa das ANE transitam por dentro de seus balanços - a quebra de uma dessas instituições pode provocar um choque capaz de frustrar um volume expressivo de gastos que afete não só os clientes da instituição, mas também seus credores, empregados e fornecedores. Além disso, em um contexto de elevada fragilidade financeira, outros atores serão contagiados pelo processo, vítimas da evaporação da liquidez e do retraimento dos mercados financeiros, multiplicando o choque inicial. Todos correrão o risco de, simultaneamente, não conseguirem atender a suas respectivas restrições de sobrevivência.

Assim, o diagnóstico minskyano distingue-se radicalmente da visão de autores que centram o problema da crise financeira na existência de fenômenos como manias ou euforias (Kindleberger e Aliber, 1992). Na visão de Minsky, a redução das margens de segurança adotadas pelos bancos na retomada do ciclo de negócios é uma decisão racional: os bancos conhecem seus clientes melhor do que o resto do mercado financeiro e reagem ao aumento de sua classificação de crédito devido à melhoria nos dados de negócios. Como afirma Kregel (2008:25): "bankers are neither gullible nor irrational".

Ademais, uma crise sistêmica geralmente não ocorre por causa das falhas de um único banco, movidas pela incompetência e a ganância de gerentes corruptos. O principal motivo é uma mudança repentina de julgamento que afeta as condições do mercado. Nessa oportunidade, um grande banco ou outra instituição financeira importante para o sistema pode subitamente não ser mais capaz de validar seus passivos e, como resultado, atender aos ajustes nas obrigações de suas contrapartes.

A única maneira de o Estado moderno conseguir evitar uma quebra bancária seguida de contágio é suspender a aplicação das penalidades associadas à restrição de sobrevivência (falência) sobre as entidades sistemicamente importantes para os mercados financeiros, particularmente os bancos. Assim, o mesmo Estado que impõe a restrição de sobrevivência tem o poder de suspender sua aplicação para determinadas empresas sempre que essa limitação se torna, do seu ponto de vista, indesejada. Foi isso o que se passou com os grandes bancos comerciais ${ }^{3}$. Para tanto, foram criadas instituições capazes de, na prática, impedir que os bancos sejam levados à falência. É o caso dos fundos garantidores de depósitos e da função de emprestador de última instância dos bancos centrais. Esses backstops do sistema financeiro moderno funcionam como gigantescos colchões de segurança sistêmicos voltados para atender discricionariamente instituições em situação adversa de caixa.

A suspensão da restrição de sobrevivência para os bancos teve, no entanto, impactos contraditórios. Tornou mais resiliente o atual sistema financeiro baseado em moeda privada fracionária. Em compensação, aumentou o incentivo a que os

\footnotetext{
${ }^{3}$ Nos últimos anos, essa isenção foi estendida a outros tipos de empresas consideradas sistemicamente importantes do ponto de vista financeiro (SIFIs).
} 
gestores dessas instituições, na prática, pudessem conviver com um nível mais baixo de margens de segurança. É um caso típico do que na literatura se chama de risco moral (moral hazard) ${ }^{4}$. Essa, por exemplo, foi uma das causas da crise de 2008: a percepção dos agentes de mercados de que o Federal Reserve System, ou seja, o principal regulador americano iria sempre resgatar o mercado após o estouro de uma bolha especulativa, o que ficou conhecido à época como o "Greenspan put" (Despeignes, 2000; Dymski, 2011).

Como contrapartida à suspensão da restrição de sobrevivência, o Estado se outorgou o direito de limitar (regular) o comportamento e as estratégias dos gestores e dos acionistas dessas instituições. Segundo Minsky:

In the absence of deposit insurance ${ }^{5}$, a bank would be subject to deposit or surveillance, and a run would occur if the bank lost the confidence of its depositors. With deposit insurance, the need for depositor surveillance is much diminished. This may mean that banks and other financial institutions will take more adventuresome positions. The central bank and the deposit insurance organization must therefore contain the exposure of banks. Regulation becomes the other side of the coin of deposit insurance. (Minsky, 1986:16)

Para preencher o vazio deixado pela suspensão da restrição de sobrevivência, os governos criaram novos tipos de limitações, específicas para os agentes financeiros regulados, por meio de indicadores contábeis, margens de capital e de ativos líquidos compulsórios. Esses atores foram submetidos à fiscalização direta e à obrigação de publicidade sobre dados e decisões, acompanhadas de penalidades para os gestores e os proprietários dessas instituições. Trata-se, portanto, de uma tentativa de emular, através de normas, o comportamento que seria esperado dessas instituições e de seus stakeholders, caso ainda estivessem sujeitos à restrição de sobrevivência. Essa visão difere radicalmente da literatura mais tradicional que vê a regulação como uma intervenção estatal para corrigir a presença de falhas de mercado ou de custos transacionais excessivos (Stiglitz, 1989; Levine, 2005).

Minsky atribui três objetivos centrais à regulação financeira. $\mathrm{O}$ primeiro seria garantir que "the payment mechanism is secure, safe and expeditious to avoid the 'contagion effect' that bankruptcy or operational risks of any commercial bank might affect the payment system" (Minsky, 1994a:6). Os perigos decorrentes do contágio dentro de um sistema muito interdependente de fluxos de caixa autônomos são considerados muito elevados sob uma perspectiva minskyana.

O segundo seria fazer com que "funds for the capital development of the economy

\footnotetext{
${ }^{4}$ Risco moral refere-se às vantagens que uma das partes de um contrato tem de adotar um procedimento que lese a outra.

${ }^{5}$ A seguradora de depósito, nesse parágrafo, refere-se à instituição que à época tinha a melhor capacidade de supervisão do sistema regulatório americano.
} 
come forth at a rate which enable as close approximation to price level stability, full employment and an acceptable economic growth to be established and sustained" (Minsky, 1994a:6). Esse objetivo decorre de uma tensão decorrente do fato de os reguladores precisarem servir, ao mesmo tempo, a "dois senhores": "one master requires assurance that the financing needed for the capital development of the economy will be forthcoming and the second master requires assurance that a safe and secure payments mechanism will be provided" (Minsky, 1994b:3). Para ele, trata-se de um dilema insolúvel que deve estar presente na mente dos reguladores e no racional das instituições que atuam nessa área:

It [...] needs to be understood now that development financing involves taking risks $[\ldots]$ The need is for a regulatory and supervising authority for the financial system that accepts that financing development opens the system to losses that have the potential for adversely affecting the safety and security of the economy's payment facilities. (Minsky, 1994b:4).

Por fim, o terceiro objetivo consiste em evitar "serious deep and long lasting depressions" (Minsky, 1994a:6). Esse tema tornou-se mais relevante na literatura após a crise de 2008. Em economias muito povoadas por agentes especulativos e Ponzi, qualquer incidente inesperado pode se transformar em um problema de graves proporções. Na medida em que a depressão se instala, a restrição de sobrevivência começa a ameaçar com a falência e a penúria de grande número de empresas e famílias. Na sua visão:

In general, economic regulation and intervention can lead to reasonably coherent behavior for an economy which, if left alone, would degenerate into incoherence. By aborting of containing incoherence, apt regulation can be constructive. (Minsky e Campbell, 1987:5)

O papel central dos reguladores financeiros deve ser o de limitar o apetite especulativo dos agentes financeiros, particularmente dos bancos, para evitar que um acidente econômico degenere em um problema de proporções sistêmicas. Entretanto, as famílias, as empresas e os bancos, na medida em que uma situação de estabilidade financeira se prolonga, são levados a experimentar e difundir estruturas financeiras que tendem a ser cada vez mais frágeis, ainda que atendam à regulação vigente.

\section{ELEMENTOS PARA UMA ATUAÇÃO REGULATÓRIA MINSKYANA}

Kregel (2014) e Wray (2017) usaram algumas das ideias de Minsky para propor uma forma moderna de "regulação dinâmica macroprudencial". Na origem da HIF existia uma preocupação de Minsky, em propor uma teoria que desse embasamento e orientação à regulação financeira. Além disso, seu intuito era levar a prática regulatória, até então voltada para aspectos microeco- 
nômicos - comportamento dos agentes -, a adotar também uma visão mais abrangente, que proporcionasse:

a more complete description of the instability of an 'economy with banking.' Such an approach needs to look behind the runs and analyze the structure of balance sheets, payment commitments and positionmaking activities. Position-making for a bank consists of the transactions undertaken to bring the cash position to the level required by regulation or bank management. In the position-making view, bank failures do not arise simply because of incompetent or corrupt management. They occur mainly because of the interdependence of payment commitments and position-making transactions across institutions and units. (Minsky e Campbell, 1987:255)

Tendo em vista a centralidade da restrição da sobrevivência, o ponto focal da regulação financeira deveria ser os balanços e os fluxos de caixa esperado dos bancos em diferentes cenários. A liquidez de qualquer agente econômico - e, portanto, sua capacidade de pagamento no futuro - depende basicamente de seus clientes conseguirem realizar os pagamentos previstos e da capacidade de os mercados de capitais poderem transformar ativos em dinheiro. Essas estimativas de fluxo de caixa, por sua vez, dependem de pressupostos de natureza macroeconômicos.

Ao mesmo tempo, os reguladores não podem abrir mão de terem um amplo conhecimento das estruturas e mecanismos de financiamento que estão sendo, a cada momento, renovados pelos mercados. Esse conhecimento é sempre um privilégio das instituições provedoras de crédito e dos gestores de riqueza. Normalmente, essa informação só se torna disponível ao público muito tempo depois que a prática especulativa foi generalizada pelo sistema financeiro e eventualmente tenha provocado uma ampla fragilização, a exemplo do que aconteceu em 2008 (Torres Filho, 2014).

A maneira de os reguladores conseguirem superar esse obstáculo, segundo Minsky, seria a realização de um processo contínuo de inspeção (examination) das instituições pelo banco central. $\mathrm{Na}$ sua opinião:

The perspective underlying the suggestions was of a dynamic, evolving set of financial institutions and relations. All too often it seems as if the Federal Reserve authorities have been surprised by changes in financial practices. One aim in the design of the examination system was to establish a regular reporting procedure which would force the authorities to be aware of institutional changes that were ongoing, and which furthermore forced the authorities to inquire into how the ongoing developments can be expected to affect the stability of the financial system. [...] One byproduct of the cash flow examination procedure will be more precise knowledge of the relations between the examined institutions and fringe banks. Such a clarification will enable the Federal Reserve to better 
know what is emerging in financial relations and to be better prepared for contingencies that might dominate as the determinants of its behavior. (Minsky, 1975:2)

A operação de um arcabouço regulatório minskyano dentro de uma perspectiva da regulação prudencial dinâmica tem três componentes importantes. O primeiro é uma teoria que explica por que o sistema monetário e financeiro está sujeito a ciclos e tende recorrentemente a crises. Problemas financeiros particulares só se tornam coletivos na medida em que a fragilização financeira já tenha adquirido um caráter mais endêmico.

O segundo é requerer uma base empírica, atualizada periodicamente por meio de fiscalizações. O sistema financeiro é institucionalmente mutante pela introdução constante de inovações financeiras movidas pela concorrência entre atores privados em busca de lucro. O conhecimento dos reguladores é limitado pelo distanciamentos temporal e institucional que têm com relação ao fechamento das operações financeiras em curso. Para tanto, a supervisão bancária precisaria, além de analisar os fluxos de caixa dessas instituições de forma prospectiva, promover recorrentemente inspeções nos bancos. Essa iniciativa permitiria aos reguladores tomar conhecimento da estrutura das operações que estão sendo realizadas, bem como poder analisar seus impactos do ponto de vista do risco individual e do sistema como um todo.

O terceiro é a centralidade e exclusividade que o Estado precisa ter na regulação financeira. $\mathrm{O}$ fato de ter, na prática, suspendido a restrição de sobrevivência das principais instituições financeiras tem como contrapartida uma atividade essencialmente indelegável. A inexistência real de uma limitação imposta por essa restrição reduziu a capacidade de o mercado exercer qualquer forma efetiva de disciplina. Assim, a sustentabilidade do processo regulatório não pode se alicerçar na ação individual ou coletiva de atores privados, como propunha, por exemplo, Greenspan (2008). Além disso, os custos financeiros, econômicos e sociais de uma crise financeira de grandes proporções não têm como ser absorvidos pelos atores privados, não importa o tamanho do capital próprio que venham a ter.

No passado recente, as discussões regulatórias evoluíram significativamente. Carvalho (2014:347) aponta que, desde o primeiro Acordo de Basileia, firmado em 1988, os requerimentos de capital, estabelecidos a partir dos ativos ponderados pelos riscos, são a principal ferramenta de regulação dos bancos. No início, a medida parecia ser um "ovo de Colombo". O intuito era frear o apetite dos bancos por ativos de maior risco - e, pressupõe-se, de maior rentabilidade - que, em contrapartida, provocavam um aumento da fragilidade financeira. Para isso, nada melhor do que obrigar os proprietários dos bancos a arcarem com o custo do risco assumido, obrigando-os a aportar proporcionalmente mais capital.

Com isso, transferia-se automaticamente a gestão do problema dos reguladores para os regulados, já que estes estariam agora alinhados na sua solução. Ao mesmo tempo, criava-se um amplo espaço de manobra para as instituições comporem o portfólio de seus ativos e operações. Desde que respeitassem a regra geral de capital 
mínimo, os supervisores não precisariam se envolver com as decisões de alocação dos gestores. O segundo Acordo de Basileia reforçou ainda mais esta abordagem, ao propiciar aos agentes que eles próprios estabelecessem os critérios de apuração dos riscos que corriam. Era uma medida embasada no espírito market-friendly que os banco centrais coletivamente só deixaram de adotar na regulação financeira após a crise de 2008.

Minsky (2008) tratou explicitamente da questão do uso regulatório dos requerimentos de capital. Na sua visão, tratava-se de um instrumento ruim para conter o efeito desestabilizador da atividade bancária, embora naquele momento específico a elevada inflação enfrentada pelos Estados Unidos se colocasse na ordem do dia. O "efeito desestabilizador", neste caso, está relacionado ao papel depressivo que o capital próprio excessivo produz na rentabilidade dos bancos que, em resposta, tentam promover a expansão do crédito. Esse comportamento alimentaria o processo inflacionário na medida em que produziria um volume de crédito acima do necessário para atender às necessidades de uma economia com estabilidade de preços.

Wray (2017) retomou esse insight de Minsky para criticar a eficácia dos requerimentos de capital, dando como evidência os fatos revelados pela crise de 2008 . Nessa oportunidade, os bancos atenderam às restrições de capital em vigor simplesmente direcionando ativos de elevado risco para suas empresas de propósito específico, extremamente alavancadas, para não serem obrigadas a registrar essas aplicações nos balanços que era necessário apresentar às autoridades regulatórias ${ }^{6}$. Em seu lugar, argumentou que, também seguindo recomendações de Minsky, as autoridades deveriam utilizar o controle direto das aplicações dos bancos, tanto no que diz respeito à concessão de empréstimos quanto à aquisição de ativos de mais elevado risco ${ }^{7}$.

O comportamento especulativo dos bancos deveria ser também inibido por meio de medidas que afetassem os métodos de avaliação de viabilidade econômica utilizados pelos bancos. Minsky era crítico contundente dos instrumentos utilizados no mercado americano, que se baseava nos ganhos esperados de capital no curto prazo, em detrimento de métodos de avaliação mais fundamentalistas baseados em fluxos de caixa e taxas internas de retorno esperados. Uma forma de tornar as estratégias especulativas mais custosas seria impor, para esses casos, maiores comprometimentos de liquidez, assegurando a manutenção de uma margem de segurança que atenda (ao menos ex-ante) à restrição de sobrevivência das instituições. Outra forma seria limitar a capacidade de fragilização das instituições financeiras, por exemplo, por meio da imposição de margens de segurança anticíclicas, que deveriam levar em conta a duração do ciclo financeiro, ou o ciclo de valorização dos ativos, e não apenas o nível da atividade da economia.

Neste sentido, o arcabouço regulatório não pode ser estático, devendo evoluir

\footnotetext{
${ }^{6}$ Para um tratamento das operações imobiliárias off-balance-sheets, ver Torres Filho e Borça (2009).

${ }^{7}$ Para Kregel (1998), inclusive, requerimentos de capital seriam instrumentos irrelevantes do ponto de vista regulatório. Ver também Carvalho (2014).
} 
tempestiva e continuamente. Até porque há outros elementos que podem colocar à prova a eficácia dos arcabouços regulatórios, principalmente as inovações financeiras. Este foi um tema tratado por Minsky apenas de uma forma indireta no que diz respeito à regulação, apesar de ser um elemento central no processo de desestabilização: as inovações seriam inexoravelmente introduzidas pelos agentes de mercado até que uma crise levasse a uma nova forma de regulação, que eventualmente proibisse as inovações mais especulativas.

Entretanto, o arcabouço minskyano oferece uma opção mais radical e intrusiva para os reguladores nesse campo. Eles deveriam estar atentos às inovações e para tanto é preciso uma supervisão in loco constante para compreender tanto quanto os atores de mercado as práticas que estão sendo generalizadas. Nada poderia estar registrado fora do balanço dos bancos se envolver negócios do interesse da instituição e dos seus controladores e gestores. Todas as operações e atividades financeiras realizadas desses atores precisariam ser reportadas e consolidadas.

Não seria demais exigir que toda inovação financeira também tivesse que ser previamente autorizada pelos reguladores, a exemplo do que acontece com a introdução de novos medicamentos. Essas novas formas de combinação de direitos, obrigações e balanços precisariam ser claramente identificadas e ter suas consequências testadas por meio de modelos que simulem seus efeitos e suas consequências sobre os agentes, as instituições e os mercados. Entretanto, é fato que muitas inovações surgem aproveitando brechas na regulação existente e dessa forma podem ser vistas como em acordo com as normas e procedimentos em vigor. Para tanto, a supervisão do regulador bancário é uma atividade essencial a ser exercida, a um mesmo tempo, com uma visão individual e sistêmica.

\section{CONSIDERAÇÕES FINAIS}

Neste artigo buscamos preencher uma lacuna na literatura explicitando que o princípio da restrição de sobrevivência, utilizado por Minsky nos primeiros anos da sua atividade profissional, é parte da sua HIF e dá origem a uma visão teórica inovadora sobre a regulação financeira. Este tema na literatura econômica é normalmente relacionado a custos de transação, falhas de mercado ou poder de mercado. A visão construída a partir das contribuições de Minsky dá ênfase a outros fatores, como a survival constraint e a HIF.

De acordo com esse conceito, todos os agentes econômicos - famílias, empresas, bancos e organismos de governo, à exceção do banco central na moeda que emite - estariam obrigados a diuturnamente ter em sua posse uma soma de dinheiro suficientemente elevada para saldar todas suas despesas correntes. A penalidade para inadimplemento pode chegar a ser a falência da empresa ou a incapacidade de uma família de atender suas necessidades mínimas.

A Restrição de Sobrevivência se integra à HIF ajudando a explicar a natureza da tipologia de risco por ele proposta - Hedge, Especulativo e Ponzi - bem 
como ao papel das margens de segurança. Segundo a leitura aqui apresentada, a regulação financeira deve ser vista como uma consequência da decisão dos Estados modernos de deixar de aplicar as penalidades da restrição de sobrevivência a um conjunto seleto de instituições financeiras sistemicamente importantes (SIFIs) e, em particular, aos bancos comerciais. O sistema monetário moderno, baseado na moeda fracionária emitida pelos bancos é intrinsecamente especulativo. Há uma contradição entre a busca do lucro e a estabilidade financeira. Ademais, a centralidade que os bancos têm para o funcionamento do sistema de pagamentos faz com que esses agentes, se levados à falência, sejam capazes de colocar em xeque a operação de todo o sistema econômico. Para evitar as consequências sociais desses episódios, os governos adotaram um tratamento diferenciado para lidar com a solvência dos bancos - bem como de instituições financeiras sistemicamente importantes. Foram criadas instituições especiais, com o objetivo de impedir que venham a ser objeto de dissolução judicial. Para isso, foram criadas a função de "banco de bancos" do banco central e as seguradoras de depósitos.

Entretanto, esse tipo de arranjo não consegue, por si só, solucionar adequadamente o problema que se propôs a resolver, uma vez que mantém intocado o objetivo de lucro dos gestores e dos proprietários das SIFIs e a dinâmica concorrencial do sistema financeiro. Assim, o óbice que se quer prevenir tende, na prática, a se agravar com a suspensão das penalidades decorrentes da restrição de sobrevivência. Esses atores, agora isentos dessas limitações, passam a deter uma capacidade ampliada de gerar lucros e, portanto, de absorver riscos - que agora passam a ser, na prática, também assumidos solidariamente pelo Estado. Para frear a exacerbação do risco moral, torna-se essencial que o governo também a obrigue SIFIs a se submeter a uma regulação financeira que lhes limite a capacidade de tomar posições demasiadamente especulativas. Essas normas precisam, portanto, promover um perfil prudente para gestores e proprietários dos bancos, emulando o comportamento que supostamente deveriam ter caso ainda estivessem integralmente sujeitas à restrição de sobrevivência.

Essa é a visão minskyana quanto à origem e a razão de ser da regulação financeira. Assim, não se trata de ser uma atividade que substantivamente busca minimizar os efeitos perversos de custos de transação, das falhas de mercado ou da incerteza. Não se trata de uma intervenção estatal em resposta unicamente à ineficiência ou à disfuncionalidade dos mercados financeiros. Diferentemente, foi uma reação necessária aos efeitos colaterais que a própria intervenção estatal gerou ao suspender a aplicação das penalidades da restrição de sobrevivência sobre determinados agentes financeiros.

A regulação não é capaz de eliminar a possibilidade de um crash de grandes proporções, mas opera para evitar que a economia seja facilmente levada a esse destino pelo funcionamento regular do sistema financeiro. Requer uma base empírica ampla e atualizada, viabilizando a atuação tempestiva das autoridades e sua 
atualização perante o avanço evolutivo das forças de mercado: a caracterização da regulação como uma atividade restrita ao Estado e, portanto, indelegável. Existe uma contradição insuperável entre o propósito de geração de lucro e competição dos atores financeiros e a estabilidade financeira. Desse ponto de vista, delegar a esses agentes a tarefa de proteger o bem comum e o patrimônio público não faz sentido, assim como não existe propósito em entregar a chave do galinheiro às raposas.

A visão minskyana oferece um edifício teórico e um arcabouço inovador para pensar a regulação no mundo real. O pensamento minskyano abre espaço para concepções mais intrusivas de regulação em termos de fiscalização e, até mesmo, da aprovação prévia pelo Estado de novos produtos financeiros, ou seja, das inovações financeiras.

\section{REFERÊNCIAS BIBLIOGRÁFICAS}

BRUNNERMEIER, M.; CROCKET, A.; GOODHART, C.; PERSAUD, A.; SHIN, H. (2009) The Fundamental Principles of Financial Regulation. Geneva Reports on the World Economy 11. Geneva/ London: International Center for Monetary and Banking Studies (ICMB)/Centre for Economic Policy Research (CEPR).

CARVALHO, F.J.C. (2014) “Can Basel III Work When Basel II Didn't?”. In: Papadimitriou, D. (Ed.) Contributions to Economic Theory, Policy, Development and Finance: Essays in Honor of Jan A. Kregel. London: Palgrave Macmillan.

CARVALHO, F.J.C. (2015) "Financial Fragility and Systemic Crises”. In: Carvalho, F. J. C. Liquidity Preference and Monetary Economies. New York: Routledge.

CASSIDY, J. (2008) The Minsky Moment. The New Yorker, 04 fev. https://www.newyorker.com/magazine/2008/02/04/the-minsky-moment.

DESPEIGNES, B. (2000) “Greenspan put' may be encouraging complacency”. Financial Times, 08 dez.

DYMSKI, G.A. (2011) “Genie out of the Bottle: The Evolution of Too-Big-to-Fail Policy and Banking Strategy in the US". Mimeo.

FREITAS, M.C.P. (2010) "Desafios da regulamentação ante a dinâmica concorrencial bancária: uma perspectiva pós-keynesiana”. Economia e Sociedade, 19(2): 233-255.

GREENSPAN, A. (2008) Statement of Alan Greenspan, House Hearing, 110 Congress, Second Session, The Financial Crisis and The Role of Federal Regulators Before The Committee On Oversight And Government Reform, October 23, Serial No. 110-209.

HERMANN, J.; PAULA, L. (2014) "Economic Development and the Functionality of the Financial System in Brazil: A Keynesian Approach”. In: Bresser-Pereira, L., Kregel, J.; Burlamaqui, L. (Eds.) Financial Stability and Growth: Perspectives on financial regulation and new developmentalism. New York: Routledge.

KINDLEBERGER, C.; ALIBER, R. (2011) Manias, panics, and crashes: A history of financial crises. New York: Palgrave Macmillan.

KREGEL, J. (1998) “The Past and Future of Banks”. Quaderni di Ricerche 21. Milano: Bancaria Editrice.

KREGEL, J. (2014) “Minsky and Dynamic Macroprudential Regulation”. Levy Economics Institute Public Policy Brief, 131. http://www.levyinstitute.org/pubs/ppb_131.pdf

KREGEL, J. (2015) “Financial Governance After the Great Recession: What Changed and What Didn't?". Revista do Serviço Público Brasília, 66 (Especial): 9-28. https://revista.enap.gov.br/index.php/RSP/ issue/view/100

LEVINE, R. (2005) "Finance and Growth: theory and evidence”. In: Aghion, P.; Durlauf, S.N. (Orgs.) Handbook of Economic Growth, Vol. 1A, Capítulo 12. Amsterdam: North-Holland

MEHRLING, P. (1999) “The vision of Hyman P. Minsky”. Journal of Economic Behavior \& Organization, 39: 129-158. 
MINSKY, H.P. (1959). Ph.D., "Bank Portfolio Determination” (1959). Hyman P. Minsky Archive. Paper 2. http://digitalcommons.bard.edu/hm_archive/2

MINSKY, H.P. (1967) "Financial intermediation in the money and capital markets". In: Pontecorvo, G., Shay, R.P.; Hart, A.G. (Eds.) Issues in Banking and Monetary Analysis. New York: Holt, Rinehart and Winston, 33-56.

MINSKY, H.P (1975) "Notes on: Suggestions for a Cash Flow Oriented Bank Examination". Hyman P. Minsky Archive, 176. https://digitalcommons.bard.edu/hm_archive/176/

MINSKY, H.P (1977) “ A Theory of Systemic Fragility”. Hyman P. Minsky Archive. 231. https://digitalcommons.bard.edu/hm_archive/231

MINSKY, H.P. (1986) “Global Consequences of Financial Deregulation”. Hyman P. Minsky Archive, 378. https://digitalcommons.bard.edu/hm_archive/378/

MINSKY, H.P. (1992a) “Reconstituting the United States' Financial Structure: Some Fundamental Issues," Levy Economics Institute Working Paper, 69. http://www.levyinstitute.org/pubs/ppb_69.pdf

MINSKY, H.P. (1992b) “The Financial Instability Hypothesis”. Levy Economics Institute Working Paper, 74. http://www.levyinstitute.org/pubs/ppb_74.pdf

MINSKY, H.P. (1994a) "Regulation and Supervision". Hyman P. Minsky Archive, 443. https://digitalcommons.bard.edu/hm_archive/43/

MINSKY, H.P. (1994b) "Issues in Bank Regulation and Supervision". Hyman P. Minsky Archive, 72. https://digitalcommons.bard.edu/hm_archive/72/

MINSKY, H.P. (2005) Induced investment and business cycles. Cheltenham: Edward Elgar.

MINSKY, H.P. (2008) Stabilizing an Unstable Economy. New York: McGraw Hill.

MINSKY, H.P.; CAMPBELL. C. (1987). "How to Get Off the Back of a Tiger, or, Do Initial Conditions Constrain Deposit Insurance Reform?” In Merging Commercial and Investment Banking-Risks, Benefits, Challenges: Proceedings, A Conference on Bank Structure and Competition, 252-66. Chicago: Federal Reserve Bank of Chicago.

NIELSON, D. (2019) Minsky. Cambridge: Polity Press (Key Contemporary Thinkers).

SOBREIRA, R. (2005) Regulação Financeira e Bancária. São Paulo: Atlas.

STIGLITZ, J. (1994) “The Role of the State in Financial Markets”. Proceedings of the World Bank Annual Conference on Development Economics 1993.

STUDART, R. (1995) "The efficiency of financial systems, liberalization, and economic development". Journal of Post Keynesian Economics, 18(2): 269-292.

TOOZE, A. (2018) Crashed: How a Decade of Financial Crises Changed the World. New York: Viking (Penguin Books).

TORRES FILHO, E.T. (2014) “A Crise do Sistema Financeiro Globalizado Contemporâneo”. Revista de Economia Política, 34(3): 433-450.

TORRES FILHO, E.T. (2019) “A Moeda em Minsky e o Atual Sistema Monetário Globalizado Americano”. Instituto de Economia da UFRJ (Texto para Discussão n ${ }^{\circ}$ 012). https://www.ie.ufrj.br/images/ IE/TDS/2019/TD_IE_012_2019_TORRES\%20FILHO.pdf

TORRES FILHO, E.T.; BORÇA, G. (2009) “As Origens e Desdobramentos da Crise do Subprime”. In: Ferreira, M.; Meirelles, B. (eds). Ensaios sobre Economia Financeira. Rio de Janeiro: BNDES.

WRAY, L.R. (2017) Why Minsky matters: an introduction to the work of a maverick economist. Princeton, N.J., Princeton University Press. 